\title{
Case Report: Long-Term Survival in Patient with Cirrhosis of the Liver and Colon Cancer K-ras Wild-Type
}

\author{
Emiddio Barletta*, Lucia Cannella, Vincenza Tinessa, Domenico Germano, Bruno Daniele \\ Department of Medical Oncology, G. Rummo Hospital, Benevento, Italy \\ Email: "emiddiobarletta@libero.it
}

Received 13 April 2014; revised 12 May 2014; accepted 11 June 2014

Copyright (C) 2014 by authors and Scientific Research Publishing Inc.

This work is licensed under the Creative Commons Attribution International License (CC BY).

http://creativecommons.org/licenses/by/4.0/

(c) (i) Open Access

\begin{abstract}
K-ras wild-type carcinoma is a tumour that is sensitive to treatment with anti-cancer and antiEGFR drugs: the combination of Cetuximab and Panitumumab with chemotherapy (Cetuximab) or as a single therapy (Panitumumab). Case Report: The clinical case presented here refers to a 68year-old patient who had been diagnosed with adenocarcinoma of the recto sigmoid with pelvic recurrence three years after surgery. The patient had a severe co-morbidity: correlated B-type liver cirrhosis. First-line chemotherapy was begun with Oxaliplatin plus Capecitabine (CAPOXI) following a relapse, and this continued for six months (six cycles), when the treatment was interrupted because of the disease's progression and hematological and gastrointestinal toxicity. Following an assessment of the K-ras, diagnosed as wild type, the patient was excluded from secondline chemotherapy treatment because of decompensated cirrhosis and the persistence of thrombocytopenia and leukopenia. The patient was put forward for biological treatment with an antiEGFR monoclonal antibody (Panitumumab). Panitumumab was administered at a dosage of 6 $\mathrm{mg} / \mathrm{kg}$ every 2 weeks for 17 months; the treatment was well tolerated, despite the cirrhosis, and the main toxicity was the skin rash. Conclusion: In patients with severe comorbidities such as cirrhosis of the liver and K-ras wild-type carcinomas, therapy with a monoclonal antibody such as Panitumumab is a treatment that is well tolerated, with few serious toxic side-effects; it also offers advantages in terms of survival and clinical benefits.
\end{abstract}

\section{Keywords}

K-ras Wild-Type Carcinoma, Metastatic Colorectal Cancer, Panitumumab, Anti-EGFR Treatment, Cirrhosis

\footnotetext{
"Corresponding author.
}

How to cite this paper: Barletta, E., Cannella, L., Tinessa, V., Germano, D. and Daniele, B. (2014) Case Report: Long-Term Survival in Patient with Cirrhosis of the Liver and Colon Cancer K-ras Wild-Type. Case Reports in Clinical Medicine, 3, 373-377. http://dx.doi.org/10.4236/crcm.2014.36083 


\section{Introduction}

Colorectal cancer represents one of the major causes of morbidity and mortality from neoplasias in all western and highly-technologically developed countries. Every year 678,000 new cases are reported worldwide, with 150,000 cases in Europe and 30,000 in Italy. At present, despite these neoplasias showing high levels of curability when compared to those in other areas of the digestive system, the five-year survival rate stands at around $40 \%$ $50 \%$, possibly reaching $80 \%-90 \%$ in the early forms. The treatment of carcinoma of the colon will depend on the stage of the disease; in stages I - III surgery is of primary importance, in stage IV surgery is only an option in selected cases. In stage IV the role of medical oncology treatment is of primary importance; chemotherapy with fluorinated pyrimidines (Fluorouracil and Capecitabine) with Oxaliplatin or Irinotecan (FOLFOX, FOLFIRI, or XELIRI or XELOX). [1] [2] are schemes that have resulted in an increase in average survival rates ranging from 6 months to more than 20. The use of anti-EGFR antibodies has demonstrated how it has been possible to achieve limited benefits, in terms of survival, in patients that were resistant to previous lines of chemotherapy including Oxaliplatin and Irinotecan. This has been demonstrated for both Cetuximab (hybrid antibody) and Panitumumab (humanized antibody) [3], but appears limited to patients who do not present mutations of the KRAS. This mutation should therefore be looked for before using these drugs [4]. Panitumumab in particular is only registered for use in patients who have already been treated with previous chemotherapies and that are EGFR positive and KRAS wild-type [5]. The K-ras oncogene is a central component of the system of signal transduction downstream of EGFR and plays a critical role in the regulation of cell growth. E was shown that the mutation status of the KRAS gene in tumor cells affects the response to cetuximab (and panitumumab), and that to benefit from treatment with these monoclonal antibodies are only the carriers of the KRAS protein not mutated (wild type) [6]. In cases where the KRAS is mutated the drug is ineffective [7]; in fact, in the presence of mutations in KRAS, which determine the constitutive activation, the proliferative signal is independent from the stimulus EGF. In these cases the block EGFR is not able to inhibit cell proliferation, making compartment the therapeutic effect of the antibody. These mutations were found in $40 \%$ of cases of cancer of the colon and rectum [8].

\section{Case Presentation}

A 68-year-old man suffering from HBV-related cirrhosis of the liver underwent a segmental resection of the sigmoid as a result of a stage pT3 pN2 M0 G2 adenocarcinoma (stage C and Koller Astler sec.). Family histori: parents died at age 70 from cardiovascular disease. In 2005 he received additional chemotherapy for 6 months with Fluorouracile + folic acid as per the Roswell Park scheme. After 3 years of follow-ups the patient presented a neoplastic recurrence at a pelvic and abdominal level, with a $35 \mathrm{~mm}$ lesion with other satellite lesions and periaortic lymphadenopathies. Tumour markers: CEA and CA19.9 normal. Not being open to new surgical treatment he was started on a course of treatment with CAPOXI, given the comorbidity (cirrhosis of the liver), the patient began a treatment with Oxaliplatin $85 \mathrm{mg} / \mathrm{m}^{2}$ every 3 weeks + Capecitabine $1800 \mathrm{mg} / \mathrm{m}^{2} / \mathrm{day} \mathrm{d} 1-14$ every 3 weeks. During the treatment he showed signs of significant hematological toxicity, in the main grade 3 thrombocytopenia (NCI) and grade 2 leukopenia (NCI). Six cycles of CAPOXI were administered and these stabilised the disease. In light of the disease stabilising, it was decided to continue treatment with Capecitabine alone at $1800 \mathrm{mg} / \mathrm{mq} / \mathrm{day}$, interrupting the Oxaliplatin because the patient had a peripheral grade 2 (NCI) neuropathy. Treatment continued until July of 2009 for a further 3 cycles. The instrumental restaging showed a slight radiological increase of the lesion, but one that could not be considered as a progression, according to the RECIST criteria. Treatment was interrupted because of the appearance of gastrointestinal toxicity, grade 2 diarrhoea and grade 2 stomatomycosis (NCI) as well as palmar-plantar erythrodysesthesia, and it was decided to proceed with a follow-up at 3 months. In October 2009 a whole body restaging CT scan (Figure 1) was performed that revealed the clear progression of the disease in the pelvic area (max. diameter of the lesion l 92mm) with the presence of intense pain in the area of the pelvis, radiating to the ipsilateral gluteus and the left leg; for this reason analgesic treatment was started with Fentanyl transdermal 25 - 50 - 100 - 125 ug. In order to programme a new line of therapy the determination of the EFGR expression and the mutational status of K-ras on the tumorous tissue of the surgical specimen was begun. The result was as follows: expression of EGFR and KRAS wild-type, and therefore predictors of a response to an anti-EGFR treatment with monoclonal Cetuximab and Panitumumab-type antibodies. Our evaluation on the choice of the best treatment for our patient was made in the wake of these data (laboratory) and given the more important comorbidity: cirrhosis of the liver-we excluded treatment with Irinotecan $+/-$ with 5-FU (a drug with a predominant liver and intestinal toxicity) and 


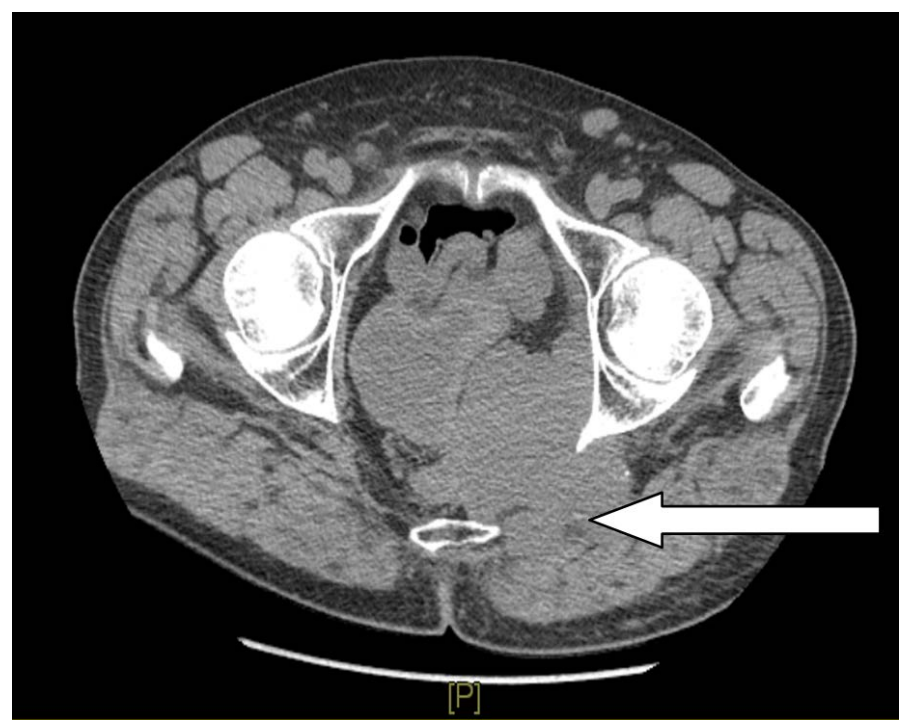

Figure 1. CT scan baseline.

taking into account the patient's blood-biochemical readings showing an increase in transaminase levels: GOT 70 U/l, ALT = 80 U/l; Leukopenia: GB = 3200; Neutrophils = 1700: Platelets = 80,000. We therefore excluded chemotherapy treatment with Irinotecan because it was hepatotoxic and opted for Panitumumab. As a consequence treatment began with Panitumumab $6 \mathrm{mg} / \mathrm{kg}$ IV every 14 days; after 2 doses a grade 3 rash (NCI) appeared, and so treatment was temporarily interrupted, postponing for another 2 weeks, and a symptomatic treatment with corticosteroids and salicylic sulphur based creams and antibiotics was ordered. At the end of the two week postponement period the rash reduced to a grade 1 - 2 (NCI). In addition to the rash, the patient also had grade 1 - 2 diarrhoea (NCI). Treatment was resumed with a grade 1 cutaneous rash, and 6 cycles were administered without the need for any interruptions and with the rash stabilising at grade 1 . After the sixth cycle a whole-body restaging CT scan with medium contrast revealed a stabilising (with a slight radiological reduction) of the pelvic mass. Treatment with Panitumumab was continued without interruption, by carrying out CT restagings every 3 months with the resultant stabilising of the disease (the pelvic lesion measured around $90 \mathrm{~mm}$ ); the treatment continued for 17 months (Figure 2). The cutaneous rash stabilised at grade 1 (NCI) in the absence of other significant toxicities.

\section{Discussion}

Currently the strategy for the treatment of metastatic colorectal carcinoma cannot take into account the evaluation of the mutational status of K-ras ref. [9]-[11], which may be present in two forms: mutated and wild-type. This evaluation allows us to identify those patients that are eligible for treatment with anti-EGFR. Approximately $40 \%$ of patients have mutated K-ras; in this case there is no inhibitory effect with anti-EGFR and therefore no benefit. $60 \%$ of patients have wild-type K-ras obtaining a clinical benefit from the use of an anti-EGFR. Panitumumab is a monoclonal humanised anti-EGFR antibody indicated as a single therapy after the failure of chemotherapies containing Oxaliplatin, Fluoropyridines and Irinotecanin in patients with K-ras wild-type metastatic colorectal carcinoma [12]. In the pivotal study, 463 patients who received multiple treatments with different lines of chemotherapy, 57\% of which were wild-type, the arm treated with Vectibix + BSC with K-ras wild-type the PFS was greater compared to BSC alone with a $(p<0.0001)$, [13] no clinical benefit in patients with mutated K-ras. Treatment with Panitumumab was usually well tolerated with a grade 3 - 4 (NCI) cutaneous toxicity of 10\%, a grade 3 - 4 (NCI) diarrhoea of 2\% and grade 3 - 4 (NCI) Hypomagnesemia of 3\% [14] [15]. The clinical case we examined was a K-ras wild-type and was treated with Panitumumab for 17 months; this produced a moderate clinical benefit (reduced pain, improved appetite, weight recovery, etc.) [16]. The predominant toxicity was cutaneous with a grade 3 - 4 rash after the first 2 doses, which then reduced and stabilised at grade 1 - 2 (NCI). Diarrhoea, on the other hand, established itself at grade 1, with short-term periods of worsening, passing to a grade 2 . Hematological toxicity was nonexistent, but periodically there was a hypomagnesemia with 


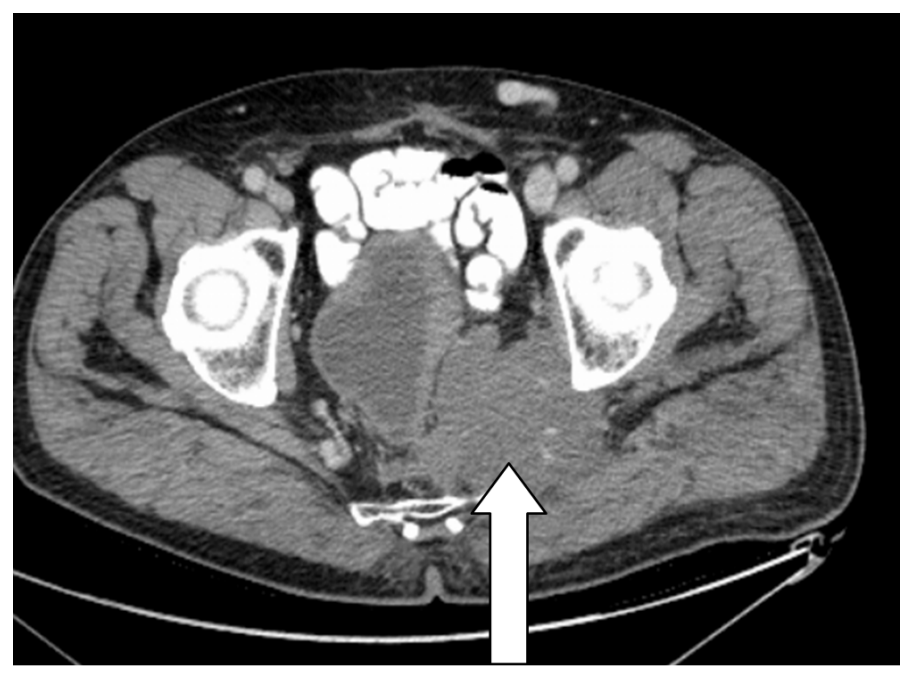

Figure 2. CT scan after 17 months.

hypocalcemia, both corrected with magnesium and calcium by os. Our patient had a PS (ECOG) $=1$, however, given that the comorbidities include cirrhosis of the liver (even if compensated ) and type II diabetes mellitus, thrombocytopenia and leukopenia (hepatopathy) and the persistence of toxicities from chemotherapy (peripheral neurotoxicity from Oxaliplatin), it was decided not to treat with chemotherapy + anti-EGFR (Cetuximab) [17]-[20], but only with an anti-EGFR: Panitumumab, which in the years from 2009 to 2011 had a therapeutic indication in single therapy after the failure of different chemotherapy regimens . The patient died in July 2013.

\section{Conclusion}

In the clinical case examined the patient in question was cirrhotic (cirrhosis is an important comorbidity that significantly limits chemotherapy treatments) and with a recurrent inoperable carcinoma of the colon (negative prognosis), maintained a stability of the disease for 17 months in succession, and overall survival of 8 years, with a humanised anti-EGFR antibody (Panitumumab) which, among other things, was well-tolerated and had a toxicity that was in the main cutaneous [21]. The patient also experienced a reduction in pain in the pelvic region, with a clear-cut reduction of analgesics, having begun at the start of the treatment with $125 \mu \mathrm{g}$ of transdermal Fentanyl which was subsequently reduced to $25 \mu$ g every 72 hours and therefore a specific control over the symptoms associated with disease. In bygone times, we would probably have stopped after a first metastatic line because the toxicity induced by chemotherapy would have not allowed treatment to be continued. However, in an age of "target therapies" a Panitumumab-type monoclonal antibody allows us to have good control over the disease, with improvements in the quality of life and the survival rate of the patient; these represent two fundamental end-points in oncology. Obviously all of this is only possible in patients who are K-ras wild-type but not in mutated K-ras cases; undoubtedly, in light of recent data, there are other molecular markers such as: B-raf, $\mathrm{N}$-ras that can identify the patient best suited for anti-EGFR treatment.

\section{Consent}

For the clinical case was called informed consent.

\section{References}

[1] Cassidy, J., Tabernero, J., Twelves, C., et al. (2004) XELOX (Capecitabine plus Oxaliplatin): Active First-Line Therapy for Patients with Metastatic Colorectal Cancer. Journal of Clinical Oncology, 22, 2084-2091. http://dx.doi.org/10.1200/JCO.2004.11.069

[2] Oh, S.C., Sur, H.Y. and Sung, H.J. (2007) A Phase II Study of Biweekly Dose-Intensified Oral Capecitabine plus Irinotecan (bXELIRI) for Patients with Advanced or Metastatic Gastric Cancer. British Journal of Cancer, 96, 1514-1519. http://dx.doi.org/10.1038/sj.bjc.6603752

[3] Wisinski, K.B., Mulcahy, M.F. and Benson, A.B. (2007) Panitumumab in Metastatic Colorectal Cancer. Clinical Ad- 
vances in Hematology and Oncology, 5, 10-11.

[4] Allegra, C.J., Jessup, J.M. and Somerfield, M.R. (2009) American Society of Clinical Oncology Provisional Clinical Opinion: Testing for KRAS Gene Mutations in Patients with Metastatic Colorectal Carcinoma to Predict Response to Anti-Epidermal Growth Factor Receptor Monoclonal Antibody Therapy. Journal of Clinical Oncology, 27, 2091-2096. http://dx.doi.org/10.1200/JCO.2009.21.9170

[5] Di Fiore, F., Charbonnier, F. and Lefebure, B. (2008) Clinical Interest of KRAS Mutation Detection in Blood for AntiEGFR Terapie in Metastatic Colorectal Cancer. British Journal of Cancer, 99, 551-552. http://dx.doi.org/10.1038/sj.bjc.6604451

[6] Karapetis, C.S., Khambata-Ford, S. and Jonker, D.J. (2008) KRAS Mutations and Benefit for Cetuximab in Advanced Colorectal Cancer. New England Journal of Medicine, 359, 1747-1765. http://dx.doi.org/10.1056/NEJMoa0804385

[7] Siddiqui, A.D. and Piperdi, B. (2010) KRAS Mutation in Colon Cancer: A Marker of Resistance to EGFR-I Therapy. The Annals of Surgical Oncology, 17, 1168-1176.

[8] McNeil, C. (2008) KRAS Mutations Are Changing Practice in Advanced Colorectal Cancer. Journal of the National Cancer Institute, 100, 1667-1669. http://dx.doi.org/10.1093/jnci/djn429

[9] Amado, R.G., Wolf, M. and Peeters, M. (2008) Wild-Type KRAS Is Required for Panitumumab Efficacy in Patients with Metastatic Colorectal Cancer. Journal of Clinical Oncology, 26, 626-634. http://dx.doi.org/10.1200/JCO.2007.14.7116

[10] Lievre, A., Bachet, J.B. and Le Corre, D. (2008) KRAS Mutations as an Independent Prognostic Factor in Patients with Advanced Colorectal Cancer Treated with Cetuximab. Journal of Clinical Oncology, 26, 374-379. http://dx.doi.org/10.1200/JCO.2007.12.5906

[11] Di Fiore, F., Charbonnier, F. and Lefebure, B. (2008) Clinical Interest of KRAS Mutation Detection in Blood for AntiEGFR Terapie in Metastatic Colorectal Cancer. British Journal of Cancer, 99, 551-552. http://dx.doi.org/10.1038/sj.bjc.6604451

[12] Seront, E., Marot, L. and Coche, E. (2010) Successful Long-Term Management of a Patient with Late-Stage Metastatic Colorectal Cancer Treated with Panitumumab. Cancer Treatment Reviews, 36, S11-S14. http://dx.doi.org/10.1016/S0305-7372(10)70002-5

[13] Van Cutsem, E., Peeters, M. and Siena, S. (2007) Open-Label Phase III Trial of Panitumumab plus Best Supportive Care Compared with Best Supportive Care Alone in Patients with Chemotherapy-Refractary Metastatic Colorectal Cancer. Journal of Clinical Oncology, 25, 1658-1664. http://dx.doi.org/10.1200/JCO.2006.08.1620

[14] Lacouture, M.E., Mitchell, E.P. and Piperdi, B. (2010) Skin Toxicity Evaluation Protocol with Panitumumab (STEEP), a Phase II, Open-Label, Randomized Trial Evaluating the Impact of Pre-Emptive Skin Treatment Regimen on Skin Toxicties and Quality of Life in Patients with Metastatic Colorectal Cancer. Journal of Clinical Oncology, 28, 13511357. http://dx.doi.org/10.1200/JCO.2008.21.7828

[15] Pinto, C., Barone, C.A., Girolomoni, G., et al. (2011) Managemente of Skin Toxcity Associated with Cetuximab Treatment in Combination with Chemotherapy or Radiotherapy. Oncologist, 16, 228-238. http://dx.doi.org/10.1634/theoncologist.2010-0298

[16] Ishiyama, Y., Kotake, M. and Matsunaga, M. (2012) Two Cases of Metastatic Colorectal Cancer in Wild-Type KRAS Effectively Treated by Panitumumab. Gan To Kagaku Ryoho, 39, 1567-1570.

[17] Peeters, M., Price, T.J. and Cervantes, A. (2010) Randomized Phase III Study of Panitumumab with Fluorouracil, Leucovorin an Irinotecan (FOLFIRI) Compared with FOLFIRI Alone as Second-Line Treatment in Patients with Metastatic Colorectal Cancer. Journal of Clinical Oncology, 28, 4706-4713. http://dx.doi.org/10.1200/JCO.2009.27.6055

[18] Raymond, E., Boige, V. and Faivre, S. (2002) Dosage Adjustment and Pharmacockinetic Profile of Irinotecan in Cancer Patients with Hepatic Dysfunction. Journal of Clinical Oncology, 20, 4303-4312. http://dx.doi.org/10.1200/JCO.2002.03.123

[19] Duillard, J.Y., Peeters, M. and Siena, S. (2008) Phase III Study (PRIME) of Panitumumab with FOLFOX-4 Compared to FOLFOX4 Alone in Patients with Previously Untreated Metastatic Colorectal Cancer. Preliminary Safety Data. Journal of Clinical Oncology, 28, 153.

[20] Cunningham, D., Humblet, Y. and Siena, S. (2004) Cetuximab Monotherapy a Cetuximab plus Irinotecan in Irinotecan Refractory Metastatic Colorectal Cancer. New England Journal of Medicine, 351, 337-345. http://dx.doi.org/10.1056/NEJMoa033025

[21] Lacouture, M.E. and Melosky, B.L. (2007) Cutaneous Reactions to Anticancer Agents Targeting the Epidermal Growth Factor Receptor: A Dermatology-Oncology Persepective. Skin Therapy Letters, 12, 1-5. 
Scientific Research Publishing (SCIRP) is one of the largest Open Access journal publishers. It is currently publishing more than 200 open access, online, peer-reviewed journals covering a wide range of academic disciplines. SCIRP serves the worldwide academic communities and contributes to the progress and application of science with its publication.

Other selected journals from SCIRP are listed as below. Submit your manuscript to us via either submit@scirp.org or Online Submission Portal.
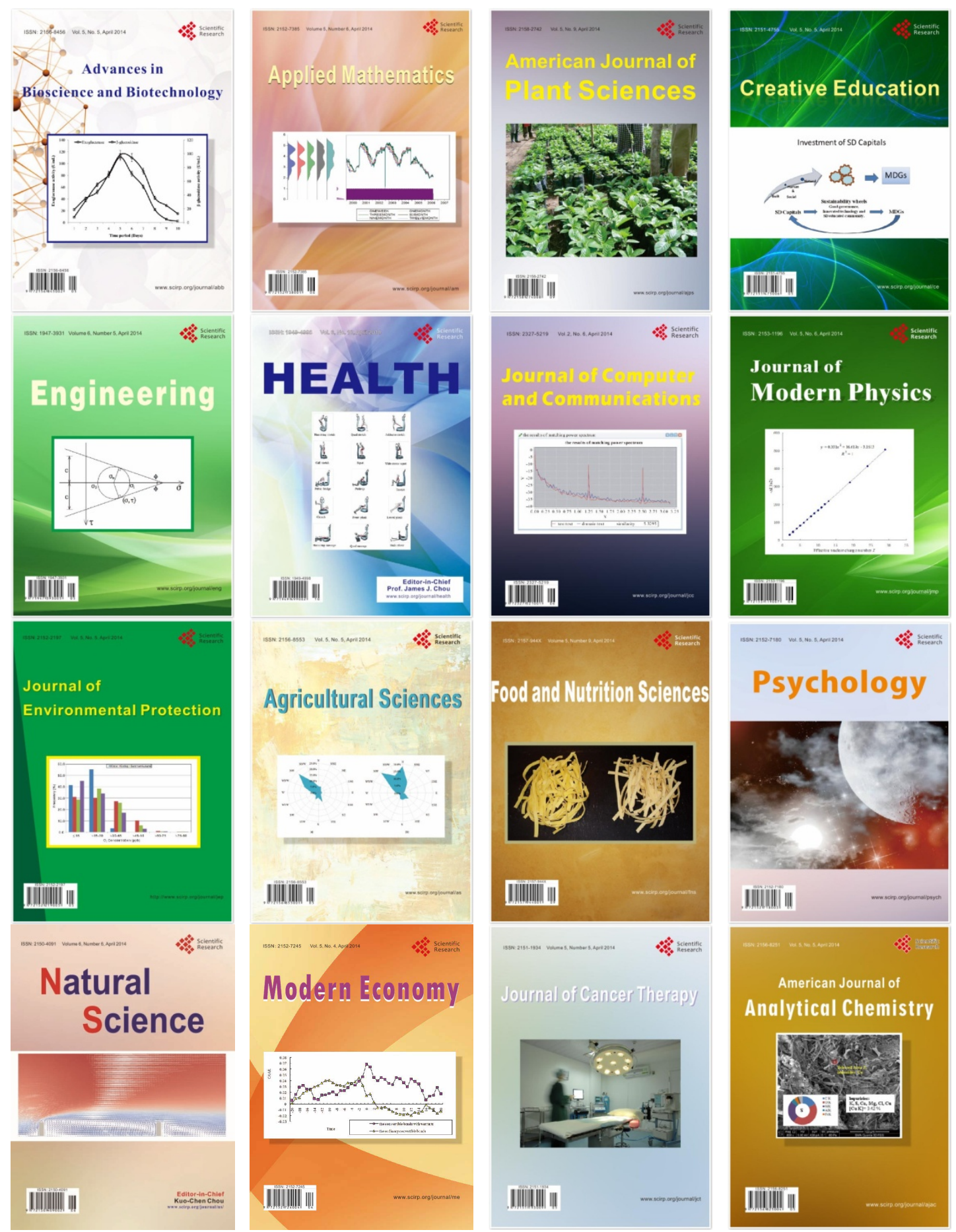